\title{
Hedgehog Signaling Governs the Development of Otic Sensory Epithelium and Its Associated Innervation in Zebrafish
}

\author{
Dora Sapède and Cristina Pujades \\ Departament de Ciències Experimentals i de la Salut, Universitat Pompeu Fabra, Parc de Recerca Biomèdica de Barcelona, 08003 Barcelona, Spain
}

The inner ear is responsible for the perception of motion and sound in vertebrates. Its functional unit, the sensory patch, contains mechanosensory hair cells innervated by sensory neurons from the statoacoustic ganglion (SAG) that project to the corresponding nuclei in the brainstem. How hair cells develop at specific positions, and how otic neurons are sorted to specifically innervate each endorgan and to convey the extracted information to the hindbrain is not completely understood. In this work, we study the generation of macular sensory patches and investigate the role of Hedgehog $(\mathrm{Hh})$ signaling in the production of their neurosensory elements. Using zebrafish transgenic lines to visualize the dynamics of hair cell and neuron production, we show that the development of the anterior and posterior maculae is asynchronic, suggesting they are independently regulated. Tracing experiments demonstrate the SAG is topologically organized in two different neuronal subpopulations, which are spatially segregated and innervate specifically each macula. Functional experiments identify the $\mathrm{Hh}$ pathway as crucial in coordinating the production of hair cells in the posterior macula, and the formation of its specific innervation. Finally, gene expression analyses suggest that Hh influences the balance between different SAG neuronal subpopulations. These results lead to a model in which Hh orients functionally the development of inner ear towards an auditory fate in all vertebrate species.

\section{Introduction}

The vertebrate inner ear is the sensory organ for hearing and balance. In the adult, it comprises a complex series of interconnected chambers, each of which hosts a specialized sensory patch that primarily mediates vestibular or auditory function. The sensory patch, the functional unit of the inner ear, consists of a sensory epithelium containing a mosaic arrangement of supporting cells and mechanosensory hair cells. Sensory afferent neurons make synaptic contact with the hair cells and then convey the extracted information to different nuclei in the hindbrain for further processing (Lewis et al., 1985; Fritzsch et al., 2000; Whitfield et al., 2002; Fritzsch and Beisel, 2004).

The sensory epithelia associated with vestibular function are the cristae and the maculae, which respectively sense angular and linear acceleration. In contrast to the vestibular apparatus that is highly conserved across vertebrates, auditory structures have evolved in different specialized endorgans such as the organ of Corti of the cochlea in mammals, or the basilar papilla in birds.

\footnotetext{
Received 0ct. 14, 2009; revised Nov. 27, 2009; accepted Jan. 20, 2010.

D.S. was a recipient of a postdoctoral Juan de la Cierva contract [Ministerio de Ciencia e Innovación (MICINN), Spain]. The work was funded by BMC2006-05604 (MICINN, Spain) to C.P. We are grateful to H. Lopez-Schier, M. Rossel, and F. Giraldez for critical reading and comments on this manuscript and to M. Linares for technical assistance. We thank to the many people who kindly provided us transgenic fish lines and reagents, especially B. Riley, C. B. Chien, C. H. Kim, S. Choy, S. H. Cheng, and S. Schneider-Maunoury. We thank F. Pinto for help with TUNEL experiments and G. Martins and M. Marx from EMBO COURSE for imaging.

Correspondence should be addressed to Cristina Pujades, Departament de Ciències Experimentals i de la Salut, Universitat Pompeu Fabra, Parc de Recerca Biomèdica de Barcelona, Dr. Aiguader 88, 08003 Barcelona, Spain. E-mail: cristina.pujades@upf.edu.

DOI:10.1523/JNEUROSC1.5109-09.2010

Copyright $\odot 2010$ the authors $\quad 0270-6474 / 10 / 303612-12 \$ 15.00 / 0$
}

The different sensory epithelia and afferent neurons of the inner ear arise from the otic placode, a simple ectodermal thickening, which is converted through extensive morphogenesis and successive steps of cell fate decisions into the mature organ (Torres and Giráldez, 1998). The development of specific inner ear structures relies on differential gene activity in spatially restricted domains of the otic vesicle at early stages. External signals influence otic patterning, and notably, Sonic Hedgehog (Shh) is required for the development of auditory structures deriving from the ventral part of the otocyst in amniotes (Riccomagno et al., 2002; Bok et al., 2005). Intriguingly, in zebrafish Hedgehog (Hh) signaling drives posterior instead of ventral otic fates (Hammond et al., 2003), indicating that the role of $\mathrm{Hh}$ in inner ear patterning may be species specific. However, hearing-specialized fish such as zebrafish use dually the saccular and lagenar maculae- two posteriorly located sensory epithelia-to mediate auditory function (Riley and Phillips, 2003). Therefore, it is possible that Hh orients functionally the development of inner ear towards an auditory fate in all vertebrate species.

In zebrafish, the utricular (anterior) and saccular (posterior) maculae are the first sensory epithelia to form in the nascent vesicle. During this period, neuroblasts delaminate from the otic vesicle to form the first-order neurons of the statoacoustic ganglion (SAG) (Haddon and Lewis, 1996). In this work, we studied in vivo the development of the otic hair cells and sensory neurons using transgenic zebrafish lines. We first describe that hair cell formation in the anterior and posterior macula is not only asymmetric but also asynchronic, suggesting they are regulated independently. We then investigated the topological organization of 
the SAG and show an anteroposterior segregation of neurons innervating each macula within the SAG. Finally we demonstrate that $\mathrm{Hh}$ signaling is required for the formation of hair cells in the posterior but not the anterior macula, and that the specific innervation patterns of the maculae are under the control of $\mathrm{Hh}$. These results highlight the importance of $\mathrm{Hh}$ pathway in the de novo formation of a fully functional sensory patch.

\section{Materials and Methods}

Zebrafish strains and maintenance. Zebrafish embryos (Danio rerio) were produced by paired mating of adult fish in the Parc de Recerca Biomèdica de Barcelona (PRBB) zebrafish facility by standard methods. AB wildtype, Isl3-GF tg(isl2b:gfp)zc7, and Brn3c-GFP strains were maintained individually as inbred lines. Brn3c-GFP line expresses GFP in the hair cells of the ear and lateral line system (Xiao et al., 2005). In Isl3-GFP (also called Isl2b) GFP is expressed in the afferent sensory neurons of the ear and lateral line system and in facial and trigeminal ganglia (Pittman et al., 2008). The $v h n f 1^{h i 2169}$ mutant line was described previously (Sun and Hopkins, 2001). All procedures used have been approved by the institutional animal care and use ethics committee (PRBB-IACUC), and implemented according to national rules and European regulations.

Cyclopamine treatment. To inhibit Hh signaling, embryos were treated with cyclopamine (CyA, LC Laboratories) (Chen et al., 2002) as previously described (Vanderlaan et al., 2005). CyA stock (10 mM 95\% EtOH; $4.12 \mathrm{mg} / \mathrm{ml}$ ) was diluted to the working concentration in tank water. Embryos were staged at 50\% epiboly/shield stage and incubated with the chorion in $90 \mu \mathrm{M}$ CyA (supplemental Fig. 1, available at www.jneurosci. org) at $28.5^{\circ} \mathrm{C}$ until the desired stage. Treated embryos were then washed several times in fresh medium before fixation or in vivo observation. Effectiveness of Hh signaling abrogation was confirmed by (1) loss of $p t c 1$ transcription, a direct target of Hh signaling that is expressed in the posteromedial part of the otic epithelium, and (2) defects in otic anteroposterior patterning (posterior expansion of the anterior marker $h m \times 3$ and reduction of the posterior marker follistatin) similar to those reported for $h h$ mutants (Hammond et al., 2003). Treatment of embryos with CyA from $60 \mu \mathrm{M}(24.6 \mu \mathrm{g} / \mathrm{ml})$ to $90-100 \mu \mathrm{M}(37.0-41.2 \mu \mathrm{g} / \mathrm{ml})$ showed that $s m u$-like $p t c 1$ inhibition was consistently obtained in embryos treated with $90 \mu \mathrm{m} \mathrm{CyA} \mathrm{(supplemental} \mathrm{Fig.} \mathrm{1,} \mathrm{available} \mathrm{at} \mathrm{www.}$ jneurosci.org). Therefore, $90 \mu \mathrm{M}$ was selected as working concentration. Embryos soaked in tank water containing the same concentrations of vehicle alone $(\mathrm{EtOH})$ did not display any phenotype and were used as control.

In situ hybridization and immunolabeling. Whole-mount in situ hybridization was performed as described previously (Hauptmann and Gerster, 1994). Probes were as follows: atoh1a, atoh1b (Millimaki et al., 2007), ptc1 (Hammond et al., 2003), zath3/neuroD4 (Park et al., 2003), gata3 (Cheng et al., 2007), ngn1, and neuroD (Itoh and Chitnis, 2001).

For immunolabeling, staged embryos were fixed in $4 \%$ paraformaldehyde (PFA) overnight $(\mathrm{O} / \mathrm{N})$ at $4^{\circ} \mathrm{C}$ and washed in PBST $(0.5 \%$ TritonX100/1\% Tween20/PBS). Embryos were generally dehydrated in $100 \%$ methanol (MetOH) at $-20^{\circ} \mathrm{C} \mathrm{O} / \mathrm{N}$ and permeabilized with Proteinase $\mathrm{K}$ (Invitrogen) at $10 \mu \mathrm{g} / \mathrm{ml}(25-35 \mathrm{hpf}$ ) or $25 \mu \mathrm{g} / \mathrm{ml}$ (48 hpf and older) at room temperature (RT) for 10-15 min. For acetylated-tubulin staining, permeabilization was performed by incubating embryos $7 \mathrm{~min}$ in acetone at $-20^{\circ} \mathrm{C}$. Afterwards, embryos were fixed for $20 \mathrm{~min}$ in $4 \%$ PFA and incubated $\mathrm{O} / \mathrm{N}$ at $4^{\circ} \mathrm{C}$ with primary antibodies in blocking solution. Primary antibodies were as follows: anti-bromodeoxyuridine (BrdU, 1:200, BD Biosciences Pharmigen), anti-phosphohistone H3 ( $\mathrm{pH} 3$, 1:300, Millipore), anti-GFP (1:200, Clontech), and antiacetylated tubulin (1:500, Sigma). After extensive washings with PBST, embryos were incubated with secondary antibodies conjugated with Alexa Fluor488 (green) or Alexa Fluor568 (red) (1:400, Invitrogen) in blocking solution at $4^{\circ} \mathrm{C} \mathrm{O} / \mathrm{N}$. After PBS washings, wholemount embryos and sections were imaged under a Leica DM6000B fluorescence microscope.

Cell cycle analyses. Cells in S phase were labeled by BrdU incorporation. Embryos staged at $24 \mathrm{hpf}$ were dechorionated and incubated for 30 min at RT in $10 \mathrm{mM} \mathrm{BrdU}$ (diluted in 1.5\% DMSO in tank water, Roche), rinsed various times in fresh tank water, and left to develop at $28.5^{\circ} \mathrm{C}$ for $30 \mathrm{~min}$. CyA-treated embryos were washed with fresh water just before BrdU incubation. Embryos were then fixed $4 \mathrm{~h}$ in $4 \%$ PFA at RT and dehydrated $\mathrm{O} / \mathrm{N}$ in $100 \% \mathrm{MetOH}$ at $-20^{\circ} \mathrm{C}$. After progressive rehydration, embryos were permeabilized with Proteinase K (Invitrogen) at 10 $\mu \mathrm{g} / \mathrm{ml} 15 \mathrm{~min}$ at RT, fixed $20 \mathrm{~min}$ in $4 \% \mathrm{PFA}$, and washed $3 \times 10 \mathrm{~min}$ in PBS. Embryos were incubated $1 \mathrm{~h}$ in $2 \mathrm{~N} \mathrm{HCl}$, washed $3 \times 10 \mathrm{~min}$ in PBS, and processed for anti-GFP and anti-BrdU immunolabeling as described above.

Cells in $\mathrm{M}$ phase were detected by anti-pH3 immunoreactivity using the above-described conditions. Embryos were transversally sectioned to localize $\mathrm{pH} 3+$ cells within different regions of the otic epithelium: anterior macula (AM), nonsensory epithelium, and posterior macula (PM).

Cryostat and vibratome sectioning. Embryos were fixed in 4\% PFA, cryoprotected in $15 \%$ sucrose, and embedded in $7.5 \%$ gelatin/15\% sucrose. Blocks were frozen in 2-methylbutane (Sigma) to improve tissue preservation, and then sectioned at $20 \mu \mathrm{m}$ thickness on a Leica CM 1510-1 cryostat. For vibratome (Leica VT1000S) sections, fixed embryos were embedded in $4 \%$ LMP-agarose before sectioning. Coronal sections of $50 \mu \mathrm{m}$ through the entire animal were obtained to have one or two consecutive sections containing SAG neurons. Transverse sections were 20-27 $\mu \mathrm{m}$ thick.

Tridimensional reconstruction of SAG. Serial transverse vibratome sections $(27 \mu \mathrm{m})$ of control and CyA-treated Isl3-GFP embryos were processed for $z$-stack imaging at the level of the SAG. 3D reconstruction of the SAGs was done using BioVis3D software.

Cell counting. Hair cell and neuron counts were performed in Brn3cGFP and Isl3-GFP embryos, respectively. For hair cells, we compiled results from several independent experiments in which we counted the numbers of GFP + cells in whole-mount living or fixed embryos (22-30 $\mathrm{hpf}$ ), or in coronal or transverse sections of fixed fish ( $34 \mathrm{hpf}-3 \mathrm{dpf}$ ). For Isl3-GFP neuron counts, $42-48 \mathrm{hpf}$ and $3 \mathrm{dpf}$ embryos were sectioned. Tissue sections were then imaged in a Leica DM6000B fluorescence microscope with DFC300KX camera under the control of LAS-AF (Leica Application Suite Advanced Fluorescence 1.8) software to perform $z$-stacks through GFP + otic ganglia (average step: $1 \mu \mathrm{m}$ ). Optical section series were processed in Photoshop 7.01 to count neurons of each optical section and determine total neuron numbers for each ganglion. The values obtained from cell counts were plotted and corresponding statistical $\mathrm{p}$ values were determined.

TUNEL assay. Distribution of apoptotic cells in the otic vesicles was determined by TdT-mediated dUTP nick-end labeling of the fragmented DNA (TUNEL, Roche). Briefly, embryos were fixed in $4 \%$ PFA O/N at $4^{\circ} \mathrm{C}$, washed in PBS, and stored at $-20^{\circ} \mathrm{C}$ in $\mathrm{MetOH}$. Following rehydration, embryos were incubated in equilibration buffer for $1 \mathrm{~h}$ at RT and then in full-strength $\mathrm{TdT}$ enzyme for $3 \mathrm{~h}$ at $37^{\circ} \mathrm{C}$. Following extensive PBST washes, embryos were blocked in $2 \% \mathrm{BMB} / 20 \% \mathrm{NGS} / \mathrm{MAB}$ and incubated $\mathrm{O} / \mathrm{N}$ with anti-fluorescein-AP (1:2000, Roche). AP activity was revealed with NBT/BCIP.

DiI and DiO tracing of inner ear afferent neurons. Larvae were fixed, transferred to petri dishes, and mounted in 1\% LMP-agarose to position them appropriately for injection. GFP fluorescence of the Brn3c-GFP line was used as a guide to visualize the hair cells contacted by afferent neurons. Neurons were then labeled by injection of DiI or DiO (Invitrogen, $1 \mathrm{mg} / \mathrm{ml}$ in DMF) directly in the anterior and/or posterior macular epithelium. Injections were performed with Femtojet microinjector (Eppendorf) under a Leica MZFLIII fluorescence dissecting microscope. From the total number of injected ears $(n=156$ for DiI injections in a single macula; $n=62$ for DiI/DiO-double injections), we obtained 64 and $48 \%$ of successful injections respectively. Injections were considered as successful when $\mathrm{Di} / \mathrm{DiO}$ fluorescence was detected at the level of central projections of otic sensory neurons (identified by the characteristic "T shape" of their ascending and descending branch in the hindbrain). Fish in which the dye extended in a sensory epithelium adjacent to the injected macula were discarded. Successfully injected fish were then processed for transverse sectioning. 


\section{Results}

Asynchronic and asymmetric development of utricular (anterior) and saccular (posterior) macula of the zebrafish inner ear

The first macular hair cells form in pairs at both ends of the nascent otic vesicle. These precocious hair cells, only present in anamniotes, were named tether cells as their kinocilia are required for otolith localization. By $22 \mathrm{hpf}$, the early-forming tether cells adopt the morphology of fully developed hair cells (Riley et al., 1997). Normally, later-forming hair cells start to accumulate soon after $24 \mathrm{hpf}$, in both the anterior and posterior macula. Tether cells and later-forming hair cells are specified in two distinct and successive phases. Their formation differentially requires the function of atoh $1 b$ and atoh 1 a respectively, two atonal homologs that have a proneural/prosensory function in zebrafish (Millimaki et al., 2007). Despite the common mechanisms leading to the formation of sensory cells in both maculae, anterior and posterior macula develops as two functionally specialized sensory epithelia.

Asymmetrical positioning of anterior and posterior macula with respect to the three axes of the otic vesicle at $3 \mathrm{dpf}$ reflects their ability to sense distinct mechanical stimulations. To better understand how this asymmetric pattern is achieved, we first characterized the dynamics of hair cell production in both maculae from $22 \mathrm{hpf}$ to $3 \mathrm{dpf}$. For this purpose, we took advantage of the Brn3c-GFP transgenic fish to follow in vivo the formation of otic hair cells, and we analyzed the time course of expression of atoh $1 \mathrm{a} / \mathrm{b}$ genes. At $25 \mathrm{hpf}, 2$ cells expressing GFP were detected at each extremity of the ears in all Brn3c-GFP embryos (Fig. $1 A, A^{\prime}$, arrowheads). In situ hybridization with atoh $1 b$ at $22 \mathrm{hpf}$ confirmed the presence of a pair of cells expressing this transcript at each pole of the ear, which identifies the early-forming tether cells (Fig. $1 E$, black arrowheads). The first differentiated laterforming hair cells were detected in the anterior macula (AM) by $27-30 \mathrm{hpf}(3.8 \pm 0.8$ cells, $n=8)$ (Fig. $1 B, B^{\prime}$, arrow) while in the posterior macula (PM) only 2 differentiated hair cells, the tether cells, were present $(1.9 \pm 0.4$ cells, $n=8)$ (Fig. $\left.1 B, B^{\prime}\right)$. From this stage onwards, the AM underwent a progressive and continuous increase in hair cell number (Fig. $1 C^{\prime}, D^{\prime}, M$ ). At $42-48 \mathrm{hpf}$, asymmetrical positioning of anterior and posterior maculae became evident (Fig. $1 C^{\prime}, C^{\prime \prime}, \mathrm{AM}$ and PM in different focal planes), and accumulation of later-forming hair cells was detected in the PM $\left(14.3 \pm 2.9\right.$ cells, $n=14$ ) (Fig. $1 C^{\prime \prime}$, arrow). Later on, at $3 \mathrm{dpf}$, both maculae have accumulated more hair cells $(\mathrm{AM}=25.3 \pm 6.0$ cells, $n=8, \mathrm{PM}=27.8 \pm 2.1$ cells, $n=8$ ) (Fig. $1 D^{\prime}, D^{\prime \prime}$ ). PM only started to accumulate later-forming hair cells at $34-36 \mathrm{hpf}(5.8 \pm$ 1.3 cells, $n=15$ ), few hours later than the AM (Fig. $1 N$ ). The normal asynchrony in hair cell production between AM and PM was prefigured by an asynchronous upregulation of atoh1a expression at the site at which each macula will later develop. Indeed, atoh $1 a$ has expanded at the anterior pole of the ear at 22-25 hpf (Fig. $1 E$, red arrowhead in the insert), a region in which the first AM differentiated later-forming hair cells were detected at 27-30 hpf (Fig. 1 B', arrow). From 27-30 hpf, a second and already asymmetric domain which intensively expressed atoh1a was established at the level of the medial wall of the otic vesicle (Fig. $1 F$, red arrowhead in the insert), preceding the detection of differentiated later-forming hair cells at $34-36 \mathrm{hpf}$ in the PM.

These data show that development of the two maculae is not only asymmetric along the three axes of the otic vesicle, but also asynchronous. This spatio-temporal uncoupling in the develop- ment of the two maculae suggests that production of their respective later-forming hair cells may be independently regulated.

\section{Requirement of Hh signaling for hair cell development in the posterior macula}

The Hh pathway appeared as a good candidate to specifically regulate saccular hair cell development, since it is required for the specification of posterior otic structures (Hammond et al., 2003). To test this hypothesis, we followed in vivo the development of hair cells in the Brn3c-GFP line from 22 hpf to $3 \mathrm{dpf}$ after abrogation of Hh signaling with CyA.

Specification and differentiation of tether cells was not affected upon blockade of the Hh pathway. Indeed, otoliths were normally positioned and $2 \mathrm{GFP}+$ hair cells were present at each pole of the ear at $25 \mathrm{hpf}$ in all treated fish (Fig. 1, compare $A^{\prime}$ with $\left.G^{\prime}\right)$. Accordingly, atoh $1 b$ expression was unchanged in CyAtreated fish at $22 \mathrm{hpf}$ (Fig. $1 E, K)$. In vivo observations and hair cell counts suggested that addition of later-forming hair cells in AM was not impaired by CyA treatment (Fig. $\left.1 G^{\prime}-J^{\prime}, M\right)$. As in control embryos, later forming-hair cells started to accumulate at 27-30 hpf (control $=3.8 \pm 0.8$ cells, $n=8$, CyA-treated $=4.4 \pm$ 0.5 cells, $n=8$ ), and then were added at a similar rate (Fig. $1 M$ ). In contrast, the posterior group of hair cells in $42-48 \mathrm{hpf}$ treated larvae not only lost its asymmetrical positioning and lay on the ventral floor of the vesicle very close to the AM (Fig. $1 I^{\prime}$ ) (Hammond et al., 2003), but also appeared consistently reduced in size (Fig. 1, compare $C^{\prime \prime}$ and $I^{\prime}$, white bracket). Detailed hair cell counts confirmed that the reduction of the posterior sensory patch was due to a dramatic decrease in hair cell numbers (control $=14.3 \pm 2.9$ cells, $n=14$, CyA-treated $=4.0 \pm 1.6$ cells, $n=14, p<0.001$ ) (Fig. $1 \mathrm{~N}$ ). Analyses of younger embryos suggested that reduction in hair cell number was likely due to incorrect specification of these sensory cells: while several later-forming hair cells were already present at 34-36 hpf in the PM of control embryos, none or very few later-forming hair cells were visualized in the posterior otic region of CyA-treated embryos (control $=5.8 \pm 1.2$ cells, $n=15$, CyA-treated $=3.3 \pm 0.9$ cells, $n=14)($ Fig. $1 N)$. Consistently, we observed a strong reduction or absence of the normal atohla expression domain at $27-30 \mathrm{hpf}$ in the posteromedial otic region of Hh signaling depleted embryos (Fig. 1, compare $F$ and $L$, inserts). The absence of Hh signaling strongly impaired further accumulation of later-forming hair cells in the posterior sensory domain. In addition, most of the $3 \mathrm{dpf}$ treated fish exhibited only one ventral macular domain (Fig. $1 J^{\prime}$ ), a malformation strikingly similar to the fused maculae of smu mutants (Hammond et al., 2003). Fusion of both sensory epithelia after CyA incubation did not allow us to assign hair cells to a specific macula. However, the total number of macular hair cells was significantly lower in CyA-treated fish when compared with control ears (control $=53 \pm 5.1$ cells, $n=8$ ears, CyA-treated $=$ $43.8 \pm 5.9$ cells, $n=5$ ears, $p<0.02$ ), probably as a result of the defective production of hair cells in the PM.

To confirm that Hh signaling was responsible for defects of the PM, we analyzed the effect of different doses of CyA on hair cell number and atoh1a expression (supplemental Fig. 1, available at www.jneurosci.org). We found that both, the number of hair cells and the expression of atohla in the PM, were reduced upon increasing concentration of CyA (supplemental Fig. 1E-I, available at www.jneurosci.org), suggesting that $\mathrm{Hh}$ regulates saccular hair cell specification by controlling the expression of atoh1a in the posteromedial otic epithelium.

Together, these results show that Hh signaling is crucial for the formation of the posterior macula, whereas the development 

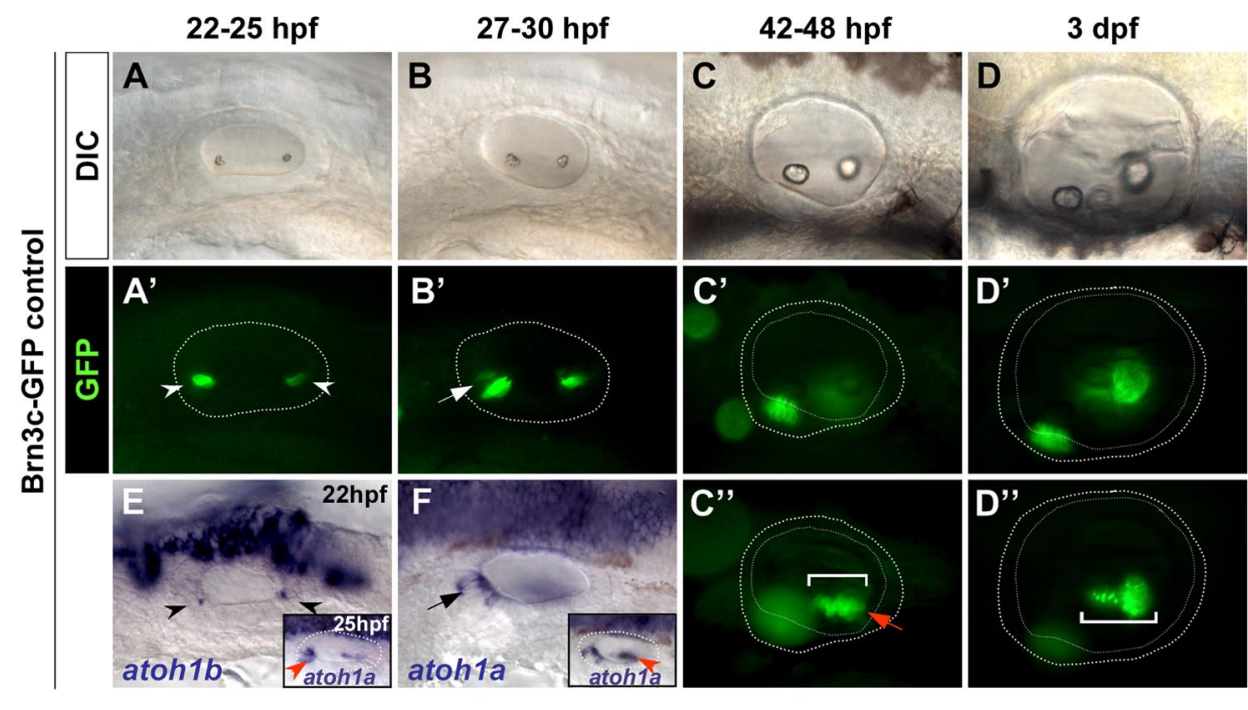

\section{ANTERIOR / UTRICULAR MACULA}
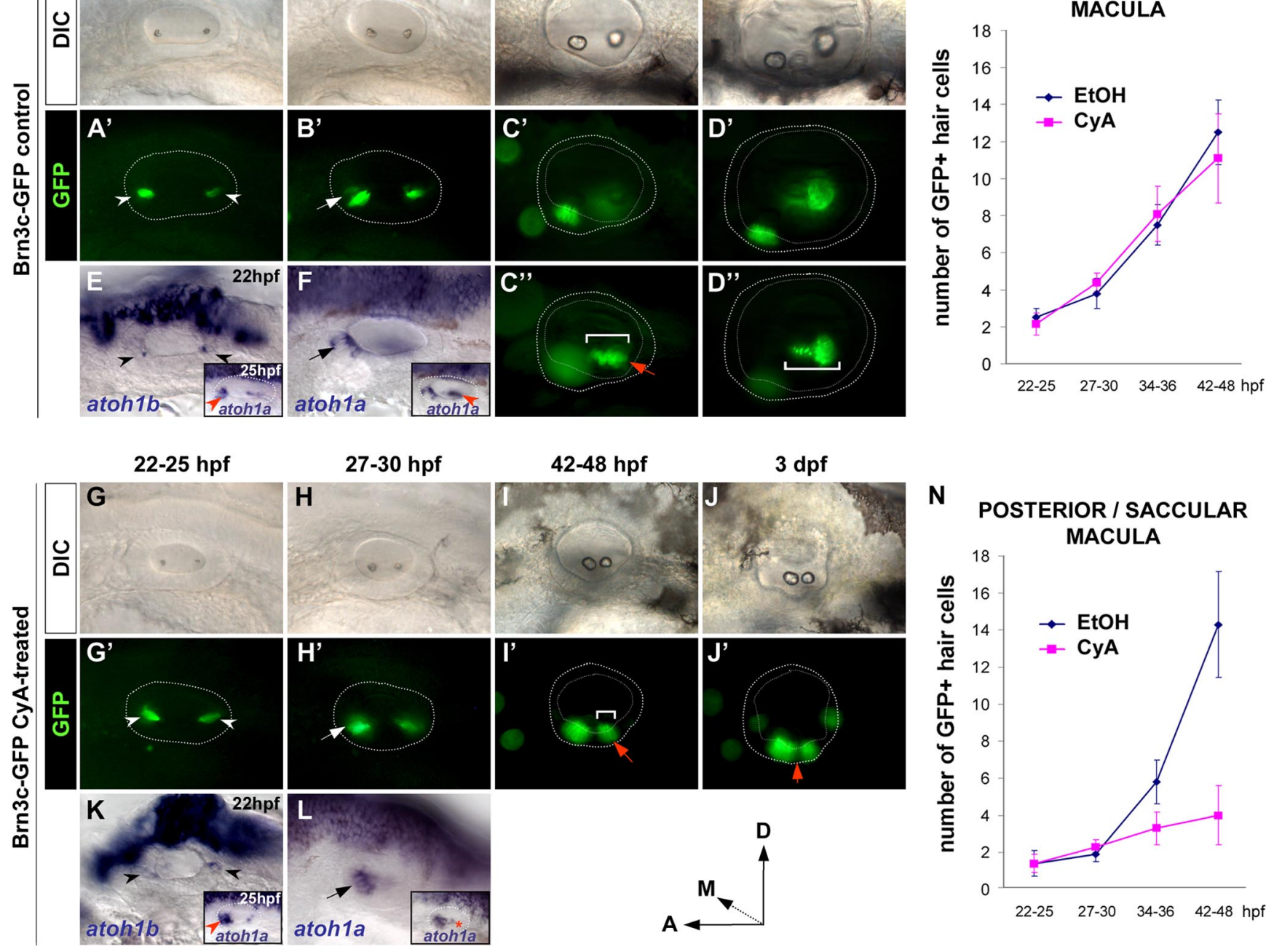

Figure 1. Hh signaling is required for the development of the posterior macula in zebrafish. $A-F$, Development of anterior and posterior maculae is asynchronic and asymmetric. $A-D, D I C$ images. $\boldsymbol{A}^{\prime}-\boldsymbol{D}^{\prime \prime}$, Corresponding fluorescence of the same otic vesicle in Brn3c-GFP transgenic fish. $\boldsymbol{A}, \boldsymbol{A}^{\prime}$, At $22-25 \mathrm{hpf}$, precocious tether cells are already differentiated (arrowheads). $\boldsymbol{B}, \boldsymbol{B}^{\prime}$, From $27-30 \mathrm{hpf}$ later-forming hair cells start accumulating in the AM (arrow). $C_{-} C^{\prime}$, Asymmetric positioning of the two maculae is evident at $42-48 \mathrm{hpf}$ as they are visualized in different focal planes ( $\boldsymbol{C}^{\prime}$ for $\mathrm{AM}, \boldsymbol{C}^{\prime \prime}$ for PM). At this stage, the PM contains several later-forming hair cells (arrow in $\boldsymbol{C}^{\prime \prime}$ ). $\boldsymbol{D}-\boldsymbol{D}^{\prime \prime}$, At $3 \mathrm{dpf}$, both AM and PM have accumulated more hair cells (compare white brackets in $\boldsymbol{C}^{\prime \prime}$ and $\boldsymbol{D}^{\prime \prime}$ ). $\boldsymbol{E}$, atoh $1 b$ expression in the early-forming tether cells (black arrowheads). At 22-25 hpf, atoh $1 a$ has expanded in the anterior otic region (red arrowhead in the insert) whereas only few isolated cells expressing this transcript can be detected in the posterior otic region from $25 \mathrm{hpf}$ (see insert). $\boldsymbol{F}$, At $27-30 \mathrm{hpf}$, atoh1a is still strongly expressed in the anterior otic region (black arrow), and its expression intensifies in the posteromedial domain (red arrowhead in the insert). $\mathbf{G}-\mathbf{L}$, Macular development in CyA-treated fish. $\mathbf{G}-\mathbf{J}, \mathbf{D I C}$ images. $\mathbf{G}^{\prime}-\boldsymbol{J}^{\prime}$, Corresponding fluorescence of the same otic vesicle in Brn3c-GFP transgenic fish after CyA treatment. $\mathbf{G}, \boldsymbol{G}^{\prime}$, At $22-25$ hpf, tether cells are formed as in control (arrowheads), consistent with the normal atoh $1 b$ expression $(\boldsymbol{K})$. $\boldsymbol{H}, \boldsymbol{H}^{\prime}$, From 27-30 hpf AM accumulates later-forming hair cells (arrow) as prefigured by normal upregulation of atoh $1 a$ expression at $22-25 \mathrm{hpf}$ ( $\boldsymbol{K}$, red arrowhead in insert). $\boldsymbol{I}-\boldsymbol{I}^{\prime}$, Asymmetric positioning of the two hair cell groups is never achieved in CyA-treated larva, and both are visible at the same focal plane. The posterior sensory epithelium (red arrow) is reduced in size (compare white brackets in $I^{\prime}$ and $C^{\prime \prime}$ ).J-J", At 3 dpf a single macular domain covers the ventral floor of the vesicle since hair cells form ectopically between the initial macular domains (red arrow in $J^{\prime}$ ). $L$, atoh $1 a$ posteromedial domain is absent (red asterisk in the insert). Dotted lines depict the contour of otic epithelium. All images are lateral views with anterior to the left. $M, N$, Time course of hair cell production in AM $(\boldsymbol{M})$ and PM $(\boldsymbol{N})$ in control (blue) and CyA-treated (purple) fish. Data from hair cell counts in the Brn3c-GFP transgenic line.

of the anterior macula is independent of Hh activity. These findings suggest that the development of each macula is under the control of different regulators. In line with this idea, a previous study showed that pax5 inactivation results in the defective formation and/or maintenance of the anterior macula and altered vestibular function, without affecting the posterior macula (Kwak et al., 2006).

\section{Hh signaling contributes to the proper development of} the SAG

Each sensory epithelium is innervated by bipolar sensory neurons that convey the information to central nuclei in the hindbrain.
Afferent otic neurons derive from the ventral floor of the otic vesicle from which neuroblasts start to delaminate at $22 \mathrm{hpf}$, and then coalesce to form the SAG beneath the otic epithelium. This process is thought to be completed by $48 \mathrm{hpf}$, as foci of delaminating neuroblasts are no longer visible within the otic epithelium (Haddon and Lewis, 1996). In Shh ${ }^{-1-}$ mice, cochleo-vestibular ganglion (CVG) fails to form as a consequence of the reduction of otic neuronal progenitors (Riccomagno et al., 2002). To address whether Hh signaling contributes to SAG formation in zebrafish, we inhibited this pathway in Isl3-GFP transgenic fish. We could detect the SAG in 26-36 hpf CyA-treated embryos (Fig. 2A-D and data not shown) in line with a report showing that specifica- 

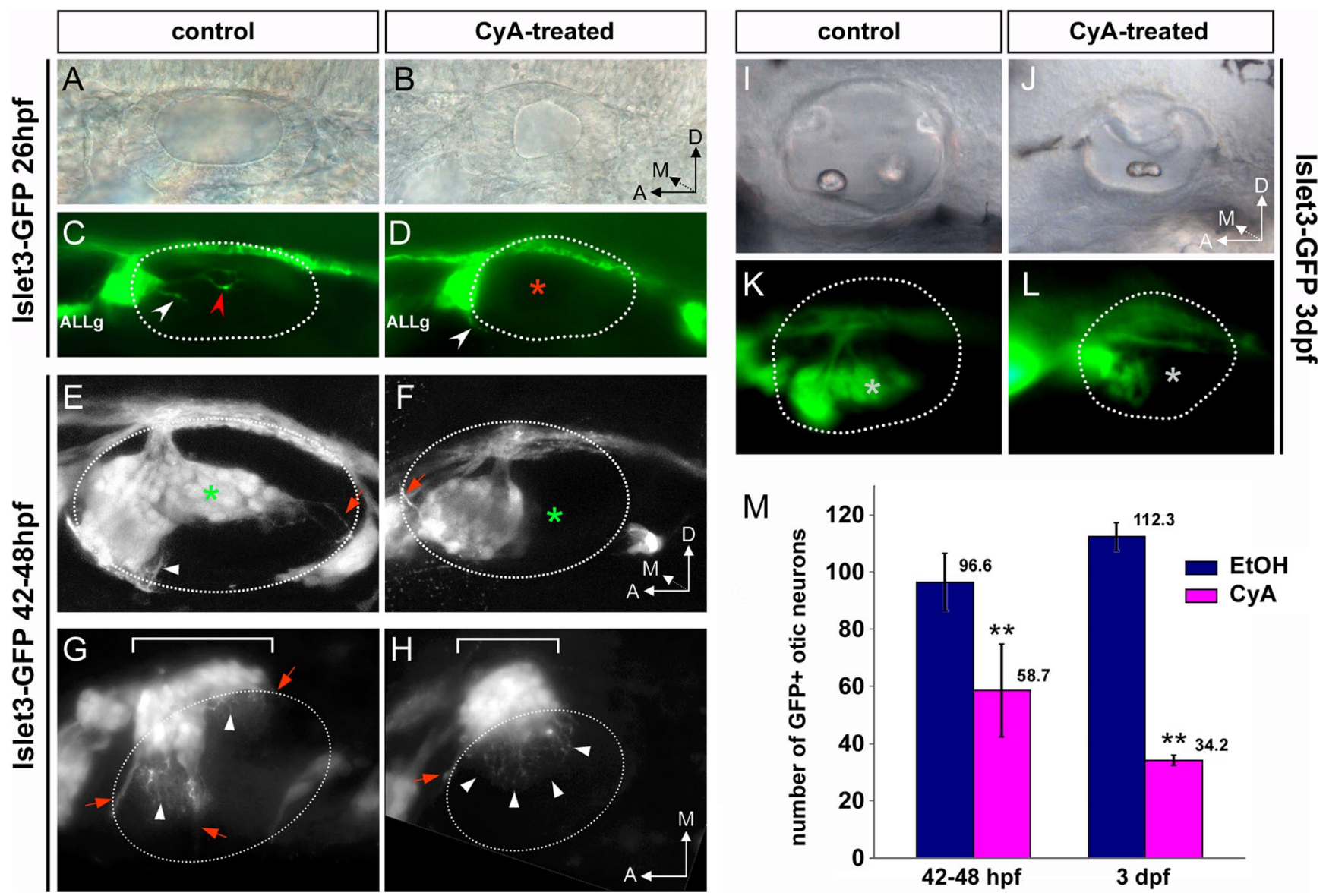

Figure 2. Quantitative and qualitative defects during the formation of the SAG in CyA-treated larvae. $A-L,|I| 3-G F P$ control $(A, C, E, G, I, K)$ and CyA-treated $(B, D, F, H, J, L)$ fish at indicated stages. $A-D, A t 26 \mathrm{hpf}, S A G$ processes are extending towards the anterior (white arrowhead) and posterior (red arrowhead) sensory patch in control embryo $(\boldsymbol{C})$, and fibers extending towards posterior sensory regions were not found in treated embryos $(\boldsymbol{D})$. $\boldsymbol{E}, \boldsymbol{F}$, Lateral views, maximal projections of confocal $z$-stacks of the $S A G$. $\boldsymbol{G}, \boldsymbol{H}$, Coronal sections containing the SAG, maximal projections of $z$-stacks. The SAG is reduced in size (compare the lengths of white brackets in $\boldsymbol{G}$ and $\boldsymbol{H}$ ) and lost its more posterior part (green/white asterisks in $\boldsymbol{E}$ and $\boldsymbol{F} / \boldsymbol{K}$ and $\boldsymbol{L}$ ) after inactivation of $\boldsymbol{H h}$ signaling. Lateral views in DIC optics $(\boldsymbol{A}, \boldsymbol{B}, \boldsymbol{I}, \boldsymbol{J})$ and fluorescence $(\boldsymbol{C}, \boldsymbol{D}, \boldsymbol{K}, \boldsymbol{L})$ of ears of fixed $26 \mathrm{hpf}$ and live $3 \mathrm{dpf}$ IsI3-GFP embryos. Dotted white lines in $\boldsymbol{C}-\boldsymbol{H}$ and $\boldsymbol{K}$ and $\boldsymbol{L}$ indicate the contour of otic vesicle. Red arrows in $\boldsymbol{E}-\boldsymbol{H}$ indicate fascicles of $S A G$ peripheral processes extending toward the developing cristae, whereas white arrowheads point to individual dendrites innervating the maculae. In $\boldsymbol{G}$ and $\boldsymbol{H}$, contrast was enhanced in the region within the dotted line, to distinguish individual processes above the otic epithelium. Note that only peripheral processes to the anterior crista fasciculate as normal in CyA-treated fish ( $\boldsymbol{H}$, red arrow), whereas individual dendrites extend to the whole ventral otic epithelium ( $\boldsymbol{H}$, white arrowheads). $\boldsymbol{M}$, Neuron counts in the SAG of $42-48 \mathrm{hpf}$ and $3 \mathrm{dpf}$ fish after inactivation of Hh signaling (purple), compared with control (blue). ALLg: Anterior lateral line ganglion.

tion of otic neuroblasts does occur in $h$ hebrafish mutants (Hammond et al., 2003). This result suggests that otic neurons are indeed specified and produced during this period. However, the analysis at $26 \mathrm{hpf}$ already revealed defects in the extension of peripheral projections towards the posterior end of the otic vesicle in treated embryos (Fig. 2A-D). As sensory neurons for inner ear and anterior lateral line are developing in extreme proximity at this stage, the number of SAG neurons could not be assessed. Therefore, this defect could result from the absence of neurons normally extending their peripheral processes posteriorly, or by impairment of the extension process. Later on, at $42-48 \mathrm{hpf}$, CyA-treated embryos displayed consistent defects in the SAG architecture: (1) the posterior part of the SAG was severely reduced (Fig. 2E,F, asterisk), and (2) the size of the SAG was smaller (Fig. $2 E-H$ ). When we counted the number of GFP+ cells in otic ganglia of Isl3-GFP larvae, the average number of GFP + otic neurons was reduced by $40 \%$ upon CyA treatment (Fig. $2 M)($ control $=96.5 \pm 10.1$ cells, CyA-treated $=58.7 \pm$ 16.2 cells, $p<0.001 ; n=11$ ganglia). To rule out the possibility that the defect induced by CyA reflected a delay in SAG formation, treated embryos were allowed to develop until $3 \mathrm{dpf}$, well after neuroblasts had completely delaminated from the otic epithe- lium. As shown in Fig. $2 I-L$, the size of the SAG in 3 dpf CyA-treated fish was also reduced. Indeed, the number of neurons in the otic ganglion was decreased by $70 \%$ (Fig. $2 M)$ (control $=112.3 \pm$ 4.8neurons, $n=4$ ganglia; CyA-treated $=34.2 \pm 1.9$ neurons, $n=5$ ganglia; $p<0.001$ ). The additional decrease in the number of SAG neurons at $3 \mathrm{dpf}$ corresponds to the loss of $40 \%$ of the otic neurons remaining in $48 \mathrm{hpf}$ treated animals.

In conclusion, Hh signaling inactivation results in a dramatic reduction in the number of SAG neurons between 42 hpf and 3 dpf.

\section{Analysis of apoptosis and cell proliferation of the inner ear} structures upon abrogation of $\mathrm{Hh}$ signaling

Our previous observations suggest that the reduction of the SAG can be attributed, at least partially, to a loss of otic neurons. To know whether the reduced number of neurons in treated fish result from increased cell death, we examined apoptosismediated cell death in otic structures (Fig. 3). There is very little cell death during development of the zebrafish otic vesicle (Bever and Fekete, 1999; Cole and Ross, 2001). In agreement with these data, very few TUNEL-positive cells were found in control larvae between 36 and 48 hpf (Fig. 3A). However, treated embryos con- 
Apoptosis - 36-42hpf

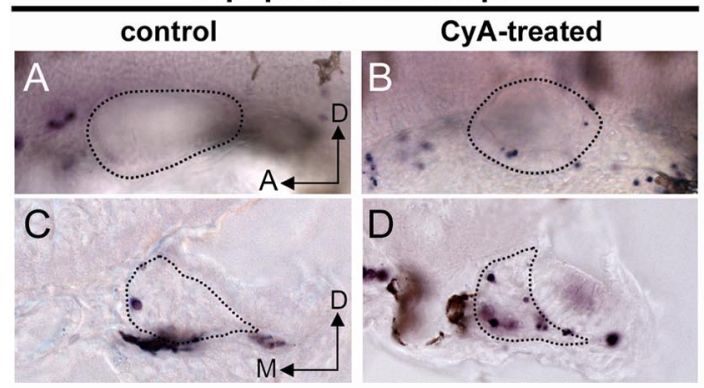

Brn3c-GFP CyA-treated - 48hpf
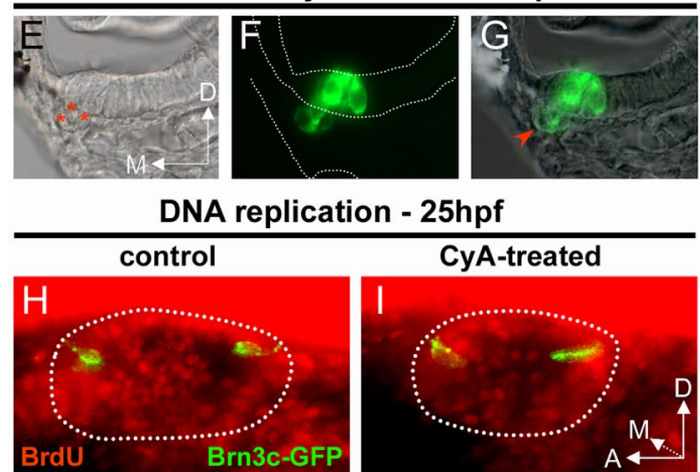

hpf
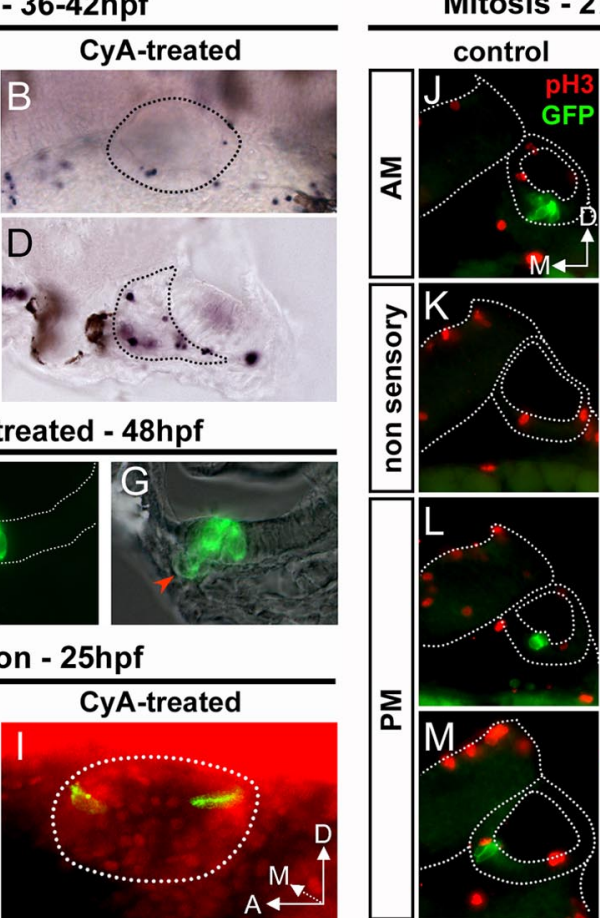

Figure 3. Cell proliferation of inner ear structures upon Hh signaling abrogation. $\boldsymbol{A}-\boldsymbol{D}$, TUNEL assay in control and CyA-treated fish at $36-42 \mathrm{hpf}$. $\boldsymbol{A}, \boldsymbol{B}$, Lateral views of the otic vesicle (dotted black line). $\boldsymbol{C}, \boldsymbol{D}$, Transverse sections showing the otic epithelium and the SAG indicated by dotted lines. $\boldsymbol{E}-\boldsymbol{G}$, Transverse section of an ear at the level of the PM in $48 \mathrm{hpf} \mathrm{Brn3C-GFPCyA-treated} \mathrm{fish:}$ DIC optics $(\boldsymbol{E})$, fluorescence $(\boldsymbol{F})$, and merged $(\boldsymbol{G})$ images. Three GFP + hair cells (asterisks in $\boldsymbol{E}$ and arrowhead in $\boldsymbol{G}$ ) are extruded from the otic epithelium to the underlying mesenchyme. Note that the sensory epithelium appears monolayered, suggesting a defect in the maintenance of hair cell/supporting cell organization in the posterior sensory domain of treated fish at $48 \mathrm{hpf} . \mathbf{H}, \boldsymbol{I}$ Lateral views of control and CyA-treated Brn3C-GFP embryos after anti-BrdU staining (red). J-Q, Anti-pH3 staining (red) in control $(\boldsymbol{J}-\mathbf{M})$ and CyA-treated $(\boldsymbol{N}-\mathbf{Q})$ Brn3c-GFP embryos. Transverse sections of the ears were ordered from anterior (top) to posterio (bottom). Note that differentiated hair cells in green do not divide or incorporate BrdU. AM: Anterior macula; PM: posterior macula.

sistently displayed increased number of TUNEL-positive cells (Fig. 3B). Transverse sections of the specimens after TUNEL assay confirmed that excess of dying cells detected in CyA-treated fish were located within the SAG and the otic epithelium between 36 and $42 \mathrm{hpf}$ (Fig. 3C,D). This stage corresponds to the period when later-forming hair cells normally differentiate in the PM, suggesting that otic neuron survival could be affected secondary to the absence of their target hair cells in treated embryos, as reported for Atoh1 null mice (Fritzsch et al., 2005). The increased cell death occurring at 36-42 hpf after CyA treatment might be, at least partially, responsible for the reduction in the number of otic neurons detected at 42-48 hpf. However, CyA-treated fish lost $40 \%$ of their SAG neurons between $48 \mathrm{hpf}$ and $3 \mathrm{dpf}$, although increased apoptosis within SAG was transient (data not shown). Because clearance of apoptotic cells is very fast in zebrafish (Bever and Fekete, 1999; Cole and Ross, 2001), the absence of apoptotic SAG neurons at this stage probably indicates that these cells undergo cell death during acute phases. Concerning the pattern of apoptotic cells in the otic epithelium, we observed that in CyA-treated embryos PM hair cells were extruded in the underlying mesenchyme (Fig. $3 E-G$, red arrowhead). Extrusion of hair cells from the otic sensory epithelium has been described as a process of elimination of apoptotic sensory cells in zebrafish mutants (Haddon et al., 1998; Kwak et al., 2006). This result suggests that Hh signaling could also be involved in the maintenance of the PM.

To further investigate the mechanism leading to the reduced number of otic neurosensory elements in treated embryos, we
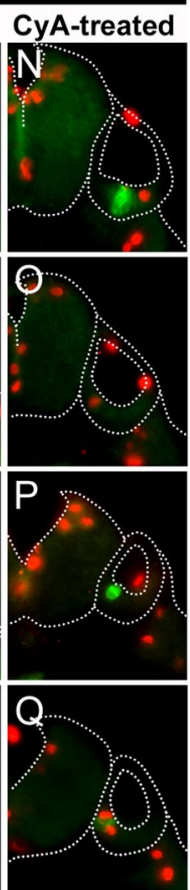

examined cell proliferation (Fig. 3H-Q). Analysis of mitotic figures using phosphohistone $\mathrm{H} 3$ revealed no significant differences between control and CyA-treated embryos regardless the otic region examined (Fig. 3J-Q). To analyze in more detail whether cell proliferation was altered in CyA-treated embryos, we performed experiments of BrdU incorporation. In control embryos, cells of the ventromedial otic epithelium incorporated BrdU whereas less incorporation was observed in the same region of treated fish (Fig. $3 H, I)$. This result is consistent with a previous study describing $\mathrm{Hh}$ as a positive regulator of cell proliferation in zebrafish (Chen et al., 2001). The decrease of cell proliferation could account for the smaller size of the otic vesicle after CyA treatment. The possibility that the lack of space within the otic vesicle of treated embryos could impair the development of the PM appeared however unlikely, as other mutants with small otic vesicles, such as $v h n f 1^{h i 2169}$, had laterally displaced but otherwise well developed PM (Lecaudey et al., 2007; supplemental Fig. 2, available at www. jneurosci.org), which correlates with atoh1a expression in the posterior end of the otic epithelium (supplemental Fig. 2, available at www.jneurosci.org).

We concluded that altered cell proliferation in the otic epithelium of treated embryos is not sufficient to account for the absence of the PM. Some reports had suggested that delaminating neuroblasts may undergo additional cell divisions before they differentiate and express neuronal specific markers (D’Amico-Martel, 1982; Fariñas et al., 2001) whereas some otic neurons may become postmitotic inside the otic epithelium (Ma et al., 1998). At the time when we examine BrdU incorporation, neuroblasts are delaminating from the ventromedial otic epithelium. Therefore, reduction in the levels of cell proliferation in CyA-treated embryos could also reflect an early defect in otic neurogenesis.

\section{Otic neurons are spatially segregated within the SAG}

The SAG appears composed of two main parts, one anteroventral and another posteromedial (Fig. 2E,G). Our observations suggested that the posteromedial portion of the SAG was absent in CyA-treated fish (Fig. 2E,F, asterisk), a qualitative defect in addition to the quantitative loss of neurons (Fig. $2 M$ ). This finding indicates that sensory and neural defects in CyA-treated embryos could be related. We therefore hypothesize that AM and PM are normally innervated by specific neuronal populations, which are located in the anteroventral part and the most posteromedial part of the SAG respectively.

To ascribe a topologic position within the SAG to the neurons innervating specifically each macula, we injected a lipophylic dye (DiI) in AM or in PM and examined their specific innervation pattern at $42-48 \mathrm{hpf}$ (Fig. $4 A, B$ ). For DiI injections, GFPpositive hair cells of Brn3c-GFP transgenic fish were used as a guide to visualize the afferent neurons that contact them. Injections in the AM consistently resulted in labeled neurons located beneath 
the most anterior third of the ear $(100 \%$, $n=44$ ) (Fig. $4 A$, top), whereas DiI injections performed at the level of the PM traced the cell bodies of corresponding SAG neurons adjacent to the medial wall of the vesicle $(100 \%, n=35)$ (Fig. $4 B$, top). Injected maculae were brightly stained at the site of DiI application, impairing imaging of labeled neurons in whole mount. To better visualize the organization of labeled neurons within the SAG, injected fish were sectioned transversally. Topologic positions of the labeled neurons were then mapped with respect to serial transverse sections of the otic vesicle, ordered from the most anterior to the most posterior (Fig. 4, sections 1-4). Depending on the number of labeled neurons, the frame of section, and the stage, we found that labeled neurons corresponding to injections in the AM were located in the first and/or second transverse section of the otic vesicle at the level of the injected AM (Fig. $4 A$, sections 1 and 2) $(78 \% n=21 / 27)$. On the other hand, $86 \%$ of DiI injections in the PM reproducibly resulted in labeled neurons located in sections 2-4 of the ear, corresponding to the level of PM and nonsensory epithelium separating both maculae (Fig. $4 B)(n=19 / 22)$. In conclusion, utricular neurons were mapped in the most anterior part of the SAG and saccular neurons in the posterior part of the SAG. In both types of injection, labeled dendrites contacting the other noninjected macula were never observed. Note that in several cases $(n=5 / 14)$, DiI injection in the PM resulted in the colabeling of neuronal processes extending posteriorly towards the epithelium containing the nascent posterior crista (PC) (Fig. $4 B$, section 4). This indicates that at least some neurons located in the posterior part of the SAG extended peripheral processes to both PM and PC at this stage. In summary, results from DiI injections strongly suggest that AM and PM are innervated by two distinct neuronal populations occupying preferential locations along the anteroposterior extent of the SAG.

To confirm this finding, we first performed double injections with a second lipophilic fluorescent dye ( $\mathrm{DiO})$ to assess the relative organization of these two neuronal populations (Fig. 4C). DiI was injected in the AM and DiO in the PM of Brn3c-GFP fish. In line with our previous results, we found that DiI- and DiO-labeled neurons never intermingled (Fig. $4 C$, top) $(n=19)$. Analysis of $z$-stacks upon transverse sectioning of the specimens showed that the two neuronal groups were completely segregated along the anteroposterior extent of the ganglion, even when DiI- and DiO-labeled neurons were present in the same section (data not shown). Although DiI-tracing does not allow strict labeling of all picture.
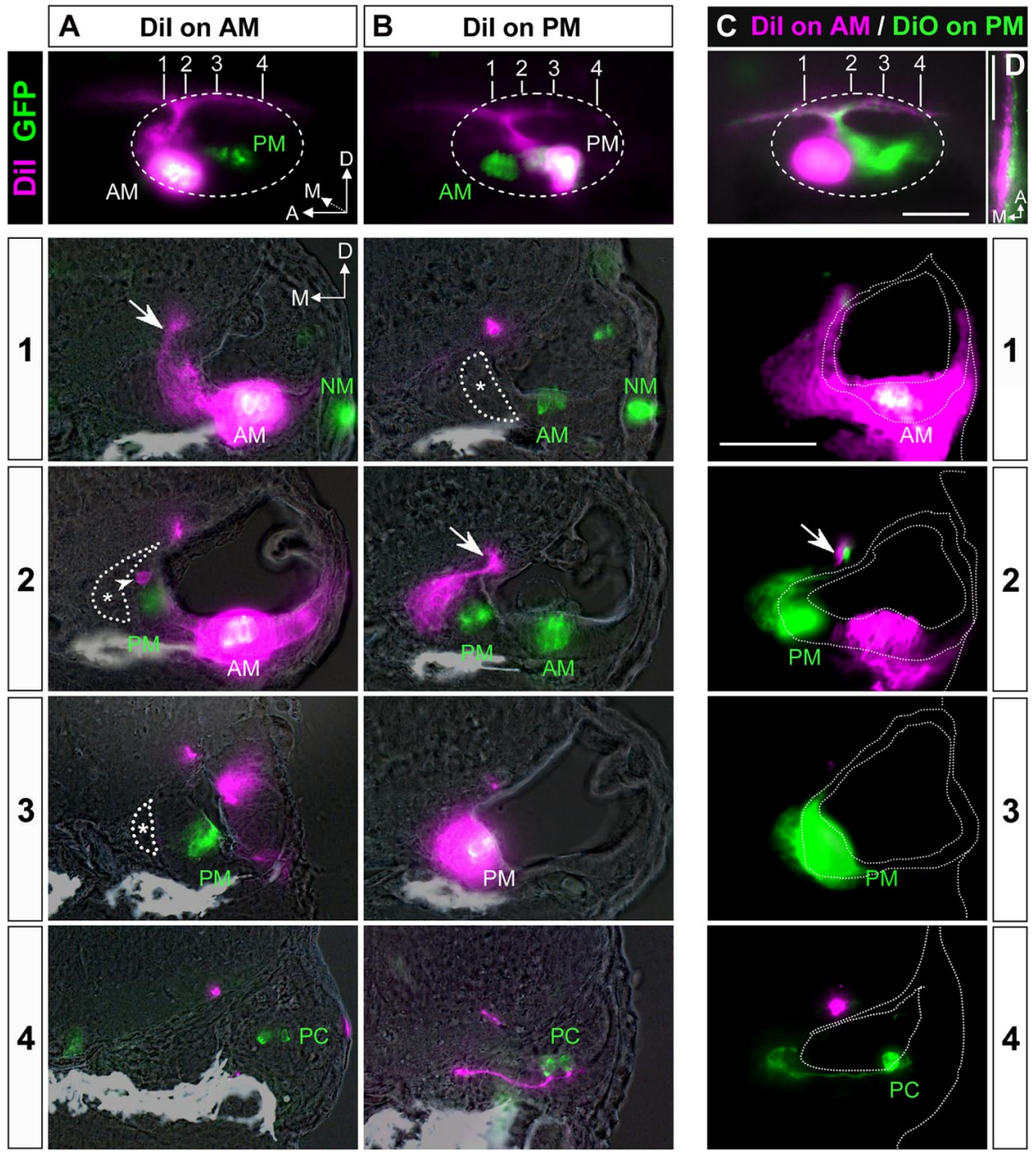

Figure 4. Neurons innervating $A M$ and PM are topologically segregated within the $S A G$ and project centrally to distinct adjacent regions. $\boldsymbol{A}, \boldsymbol{B}$, Single Dil injections in the $\mathrm{AM}(\boldsymbol{A})$ or PM $(\boldsymbol{B})$ of Brn3c-GFP fish at $42-48 \mathrm{hpf}$. Upper row shows lateral views of injected ears under fluorescence, and the level of transverse sections is indicated (ordered from 1 to 4 , anterior to posterior). Fish sectioned at equivalent anteroposterior levels allow comparing the position of utricular and saccular neurons according to the same morphological landmarks. Labeled neurons corresponding to injected AM are found in the anteriormost section of the ear at the level of the AM sensory epithelium ( $\boldsymbol{A}$, section 1$)$, whereas neurons corresponding to PM are visible in two sections beneath the medial wall of the otic vesicle and the PM ( $\boldsymbol{B}$, sections 2 and 3 ). The axons enter the hindbrain and bifurcate in ascending and descending branches at a level between sections 1 (for utricular neurons) and 2 (for saccular neurons) (arrow). Dil injection in PM can result in colabeling of neural processes extending to the posterior crista (PC in section 4). Dotted lines in the sections delineate the limits of SAG visible in DIC optics. Contrast enhancement of the purple channel in the zone in which cell bodies are located was necessary due to the very bright Dil-staining at the point of injection. Fluorescent images of section 1 for $\boldsymbol{A}$ and section 2 for $\boldsymbol{B}$ are composite images obtained by maximal projection of 2 to 3 different focal planes to see the maximal number of labeled cell bodies; idem for section 4 in $B$ and $C$ to allow the reconstitution of the entire length of neuronal processes contacting the $P C$. C, Double injection with Dil in the AM and DiO in the PM of the same ear of a 42-48 hpf Brn3c-GFP embryo. Upper row shows lateral view of whole-mount fish after injection, and the level of transverse section is indicated (ordered 1-4, from anterior to posterior). Section 2 shows the labeled central projections (see white arrow), with utricular neurons projecting more medially (Dil, purple) and saccular neurons more laterally (Di0, green). Section 4 is from another injected fish and illustrates the colabeling of PC innervation after tracing of the saccular neurons. $D$, Dorsal coronal section of a fish processed for Dil/Di0 injections, showing adjacent but distinct areas of central projections for utricular and saccular neurons. All images are fluorescent pictures of single focal plane except for section 4. AM, Anterior macula; PM, posterior macula; NM, neuromast. Scale bar, $50 \mu \mathrm{m}$; enlargement in $\boldsymbol{D}, 25 \mu \mathrm{m}$. Axes are indicated in the

the afferents corresponding to one macula, no DiI/DiO double labeled neurons were found. These double injections also highlighted some features of the specific central projection patterns of maculae: (1) axonal processes from neurons connecting each macula fasciculate in distinct bundles, the one corresponding to utricular neurons enters the hindbrain in a more medial position than the one for saccular neurons (Fig. 4C, section 2, arrow); (2) after bifurcation, the ascending and descending branch of utric- 


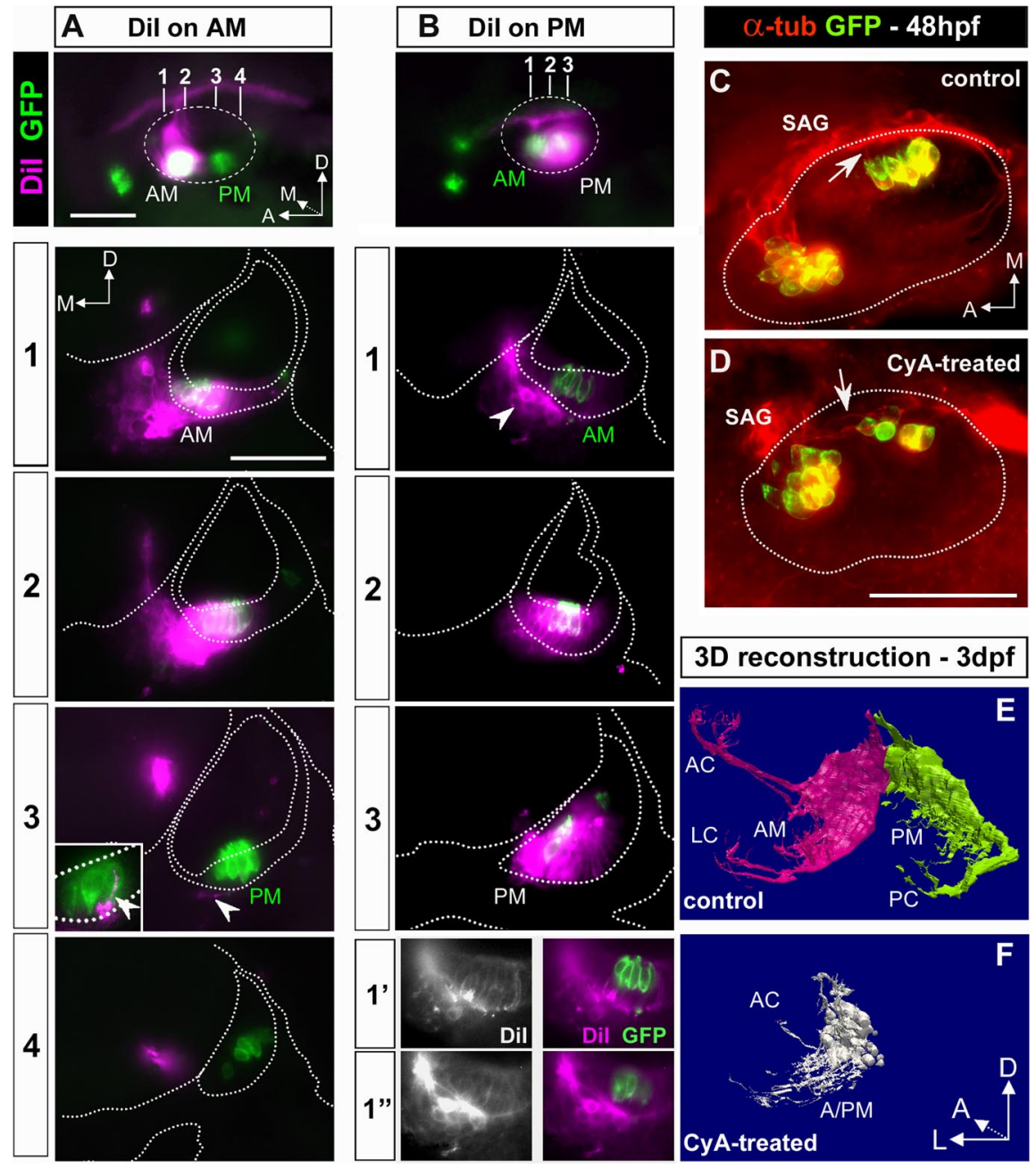

Figure 5. Inactivation of Hh signaling disrupts the specific innervation pattern of the maculae. $A, B$, Dil injections in Brn3C-GFP fish treated with CyA. Upper row shows fluorescence images of the ear in lateral views after injection. Fish were transversally sectioned and the level of the sections ordered from 1 to 4 as in Figure 4. A, Dil-labeled neurons corresponding to AM are visible in sections 1-2. SAG is reduced compared with control such as no otic neurons are present in sections $3-4$. Section 3 shows a peripheral process originating from a labeled anterior neuron (arrowhead) extending towards the remaining PM and contacting a posterior hair cell (insert, dotted lines indicate the contour of the otic epithelium). Contrast enhancement of the purple channel allows seeing the misrouted neural processes of posterior neurons innervating the AM in section 3. $\boldsymbol{B}$, Only few neurons are detected in section 1 (arrowhead) after Dil injection in the PM, and no neuron is visible in more posterior sections (sections 2-3) in contrast to control fish. The few labeled saccular neurons extend processes to the AM, as detailed in two different focal planes $\left(1^{\prime}-1^{\prime \prime}\right)$ at the level of GFP-positive cells of the AM of section 1. C, D, Dorsal views of Brn3C-GFP embryos after anti-acetylated tubulin staining (red) showing that fibers from the anterior portion of the SAG were misrouted in CyA-treated fish (arrow in $\boldsymbol{D}$ ). $\boldsymbol{E}, \boldsymbol{F}, 3 \mathrm{D}$ model of the topological organization of the SAG in control and CyA-treated IsI3-GFP fish, performed with BioVis 3D software. Images were obtained by reconstructions of the SAG volume from z-stacks of transverse sections. LC/AC/PC: lateral/ anterior/posterior cristae. Scale bar, $50 \mu \mathrm{m}$. Axes are indicated in the pictures.

ular and saccular afferent neurons keep parallel but distinct central pathways, more medial for utricular and more lateral for saccular neurons (Fig. 4D). Finally, we performed DiI injections in one of the two subgroups of otic neurons identified in the Isl3-GFP transgenic fish. Injection in the posteromedial part of the SAG resulted in labeled dendrites invading the PM, and a bundle of labeled fibers extending towards the PC. Reciprocally, injections in the anteroventral part of the ganglion, labeled peripheral processes that kept confined to the anterior sensory region (data not shown).

Altogether these experiments lead us to conclude that the SAG is topologically organized in at least two different neuronal subpopulations, which are spatially segregated at $42-48 \mathrm{hpf}$. AMspecific neurons are located anterior and ventral to the ear within the anterior part of the SAG, whereas neurons innervating PM and PC form a posterior subgroup within the SAG, which is positioned medial to the otic vesicle.

\section{Specific innervation of the maculae is} under the control of $\mathrm{Hh}$

Once we established that neurons innervating each macula were spatially segregated, the next step was to study whether this organization was under the control of Hh signaling. Using acetylated-tubulin immunostaining to visualize macular innervation, we showed that the anterior portion of the SAG was innervating the $\mathrm{AM}$, while the PM was contacted by short processes emerging from the posterior portion of the ganglion (Fig. 5C, arrow). In contrast, staining in CyA-treated fish suggested that neurons for a specific macula were not clustered together and were intermingled within the reduced SAG (Fig. 5D, arrow). To test this hypothesis we performed DiI injections in anterior or posterior macula in Brn3c-GFP embryos treated with CyA (Fig. 5A,B). Injections were done mainly at $42 \mathrm{hpf}$ because the close proximity or fusion of maculae at later stages does not allow the labeling of each sensory patch separately. DiI injected in the AM revealed labeled neuronal cell bodies all along the anteroposterior extent of the reduced ganglionic mass, suggesting that CyA treatment abolished the preferential distribution of utricular neurons in the anterior part of the SAG (Fig. 5A, sections 1 and 2), and that most of the remaining SAG neurons were innervating AM hair cells. Moreover, detailed observation revealed a labeled dendrite from a utricular neuron contacting one hair cell of the mispecified PM (Fig. 5A, section 3, insert). These results suggest that after inactivation of the Hh pathway, neurons normally innervating the AM now emit collateral fibers to the other sensory epithelium. Reciprocally, when DiI was injected in the posterior hair cell group of CyA-treated fish, few labeled neurons were found beneath the noninjected AM (Fig. $5 B$, section 1 ), and these neurons were extending collateral dendrites towards this anterior sensory epithelium (Fig. 5B, sections $1,1^{\prime}, 1^{\prime \prime}$ ). Very few neurons were labeled after PM injection in CyA-treated fish compared with control, indicating that the neuronal population innervating the PM was selectively reduced (Fig. 5B). Finally, we performed 3D reconstructions of SAGs for control and CyAtreated $3 \mathrm{dpf}$ Isl3-GFP fish. Following of individual peripheral processes, combined with the results of our tracing experiments allowed us to elaborate a model for the topological organization of the SAG. In control fish, the anteroventral portion of the SAG innervated the AM, anterior and lateral cristae (AC/LC), whereas the posterior part of the SAG innervated the PM and PC (Fig. 5E). In contrast, when Hh signaling was abrogated, the SAG was 
reduced and disorganized and morphologically resembled the anterior part of the normal otic ganglion (Fig. $5 F$ ).

These results show that spatial anteroposterior segregation of utricular versus saccular otic neurons is lost in absence of functional Hh signaling, and that remaining SAG neurons extend misrouted fibers towards the "wrong" sensory epithelium.

\section{Hh signaling influences the balance of SAG neuronal subpopulations}

We did not observe a clear reduction in the size of the SAG upon Hh inactivation before $42-48 \mathrm{hpf}$, pointing to the involvement of Hh in neuron survival more than in initial neuron production. However, it was proposed that in mouse Shh specifies the number of CVG progenitors by regulating $n g n 1$ transcription (Riccomagno et al., 2002). To test whether $\mathrm{Hh}$ has a role in the generation of otic neurons, we examined the expression of proneural and neurogenic markers in control and CyA-treated embryos. Ngn1 is the earliest neuronal determinant expressed in proliferating otic epithelial neuroblasts before and during their delamination (Ma et al., 1998; Andermann et al., 2002), and is necessary to activate downstream bHLH factors, including NeuroD, in the differentiating neuronal population (Ma et al., 1998). While $n g n 1$ is rapidly downregulated in neuroblasts after delamination, neuroD expression persists in most of the differentiating and mature otic neurons (Andermann et al., 2002). In CyA-treated fish, ngnl and neuroD expression was unchanged at $18-20 \mathrm{hpf}$ (data not shown). At 24-26 hpf, the ventromedial otic epithelium of control fish broadly expressed ngn 1 (Fig. 6A) (Andermann et al., 2002) and although CyAtreated embryos maintained strong $n g n 1$ expression levels in the anterior part of the otic epithelium, little or no expression was detected in its posteromedial region (Fig. $6 \mathrm{~A}$, asterisk). A similar change was observed in the neuroD-expressing domain (Fig. 6A, asterisk), suggesting that neuronal precursors normally produced in the posterior part of the otic neurogenic domain were ablated in the absence of functional Hh pathway.

To distinguish the neuronal population selectively dependent on Hh signaling we investigated the expression of two genes involved in the establishment of neuronal identity, neuroD4 (NeuroM/Math3) and gata3 (Fig. 6). NeuroD4, like NeuroD, is able to induce neuronal fate under ectopic expression (Lee et al., 1995; Takebayashi et al., 1997) and might cooperate with ngn1 to specify different neuronal identities in a subset of cranial ganglia in zebrafish (Park et al., 2003). We found that neuroD4 was expressed in a pattern similar to ngn 1 and neuroD (Fig. 6A), in few differentiating SAG neurons and in the neuroblasts delaminating

A

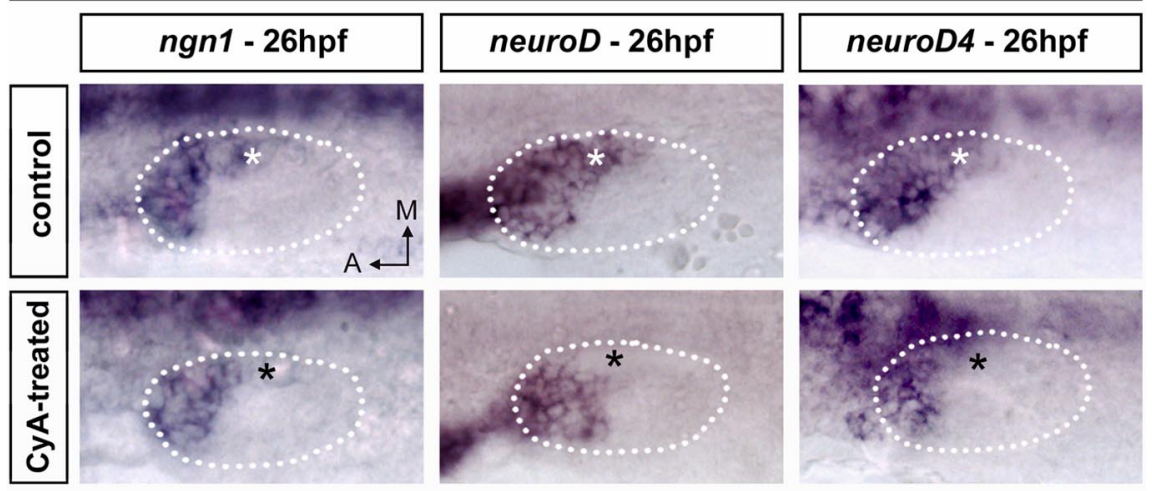

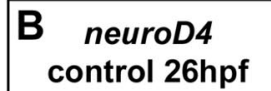

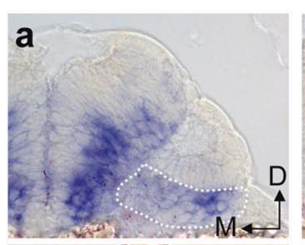

b

Figure 6. Role of Hh signaling in otic neuronal identity. $A$, Dorsal views of ngn 1 and neuroD4, and ventral views of neuroD expression in control and CyA-treated fish. ngn 1, neuroD and neuroD4 expression disappears from the posteromedial otic territory in CyA-treated fish (asterisks). Anterior is to the left. $\boldsymbol{B}, \boldsymbol{C}$, Transverse sections from anterior (a) to posterior (d) throughout the otic anterior section ( $\boldsymbol{a}$, dotted lines). Brackets in $\boldsymbol{b}$ sections indicate the broader delaminating zone. Arrowheads in $\boldsymbol{c}$ indicate the posterior part of the neurogenic domain. $\boldsymbol{D}$, Transverse sections from anterior $\left(\boldsymbol{a}, \boldsymbol{a}^{\prime}\right)$ to posterior $\left(\boldsymbol{d}, \boldsymbol{d}^{\prime}\right)$ through the otic vesicle of gata3-hybridized embryos at 32-35 hpf. The SAG extends in the two first sections ( $\boldsymbol{a}, \boldsymbol{b}$; dotted lines). Note the excess of gata3expressing neurons within the SAG of CyA-treated embryo, compared with control. from the ventral otic epithelium (Fig. $6 B a-B c$ ). However, after $\mathrm{Hh}$ signaling inactivation, the expression of these three genes was similarly reduced in the posteromedial domain of the otic vesicle (Fig. 6A, asterisk). These findings appear more consistent with the proposed function of NeuroM in chick in identifying nascent neuroblasts (Bell et al., 2008), rather than in defining a distinct neuronal identity. The other candidate, GATA3, selectively marks cochlear sensory neurons within the CVG in mouse (Lawoko-Kerali et al., 2004) and has been involved in the switch of neuronal identities, from branchiomotor to octaval efferent neurons in the hindbrain and from vestibular to auditory otic 
afferent neurons (Karis et al., 2001). We examined its expression pattern from 18-36 hpf, and found that, as in mouse, gata3 was also expressed in the inner ear (Fig. 6C,D). gata3 was broadly distributed in the otic epithelium at early stages (data not shown). This epithelial expression was restricted by $26 \mathrm{hpf}$ to the posterior and medial part of the otic epithelium with intensive expression in the cells limiting the delaminating zone (Fig. $6 \mathrm{Bb}$ ). However, the posterior end of the delaminating zone, marked by neuroD4, was comprised within the gata3-positive territory of the otic epithelium (Fig. $6 B c, C c$, arrowheads). Later on, by $32-35 \mathrm{hpf}$, while a small population of differentiating SAG neurons expressed gata3 in control embryos, CyA-treated fish displayed an increased proportion of gata3+ neurons within the SAG (Fig. $\left.6 D a, b, a^{\prime}, b^{\prime}\right)$. Although the functional significance of gata3 expression in a subset of SAG neurons in zebrafish remains unclear, as zebrafish does not have a cochlea, this result indicates that $\mathrm{Hh}$ signaling can modulate the balance among different SAG neuronal subpopulations.

\section{Discussion}

\section{Hh signaling governs the formation of the posterior macula} and associated first-order neurons

Our results show that $\mathrm{Hh}$ signaling governs the formation of a fully functional posterior (saccular) macula. But how Hh signaling can be involved in defining a PM-specific identity?

It is assumed that the development of all otic neurosensory elements crucially depends on the function of three atonalrelated bHLH transcription factors: atoh1 for hair cell formation (Bermingham et al., 1999; Millimaki et al., 2007), ngn1 for sensory neuron determination (Ma et al., 1998; Andermann et al., 2002), and neuroD for sensory neuron differentiation and survival (Kim et al., 2001).

In the zebrafish ear, the prosensory function is thought to be mediated by atohla/b genes (Millimaki et al., 2007). We showed that the posterior domain of atoh1a expression in the zebrafish inner ear appears later than the anterior domain, and is selectively affected by reducing Hh signaling. Although we cannot conclude whether this effect is direct, our results indicate that the sensory elements generated in the posterior domain of the otic vesicle are under the control of $\mathrm{Hh}$ signaling. In contrast, $\mathrm{Ngn} 1$ has been recently proposed as the proneural (prosensory) gene in mouse that defines a field of progenitors giving rise to both sensory neurons and macular hair cells, by auto- and cross-regulation with Math1/Atoh1 (Fritzsch et al., 2006; Raft et al., 2007). Shh ${ }^{-1-}$ mice have a reduced expression of $\mathrm{Ngnl}$ in the otic epithelium (Riccomagno et al., 2002). This could indicate that in mouse also, Hh signaling may induce the expression of the prosensory gene $N g n 1$ in a specific territory of the inner ear.

Concerning otic neuronal identity, fate mapping studies in chick and mouse (Koundakjian et al., 2007; Bell et al., 2008) proposed that specific neuronal identities and wiring decisions are influenced by differential temporal sorting and/or spatial origins of the otic neurons. External cues such as $\mathrm{Hh}$, may regulate within the otic epithelium the restricted expression of one (or a combination of) transcription factor(s), that direct neuronal identity upstream of $n g n 1$. In mouse, GATA3 expression in the early otic epithelium is spatially and temporally restricted to the neurogenic territory responsible for the production of cochlear neurons, and later on selectively marks the cochlear part of the CVG (Karis et al., 2001; Lawoko-Kerali et al., 2004; Koundakjian et al., 2007). Our study showed that in the zebrafish, gata3 expression domain overlaps only partially with the otic neurogenic domain. However, although the gata $3^{+}$domain coincides with the territory in which the Hh receptor $p t c 1$ is expressed, Hh does not directly regulate gata3 expression in the otic epithelium.

Similar to the otic neuronal defects described in CyA-treated fish, NeuroD ${ }^{-1-}$ mice display an increased apoptosis in otic ganglion, defects of early fiber extension, and a reduction of the peripheral innervation, affecting differentially distinct sensory epithelia (Kim et al., 2001). This suggests that neuroD acts differentially on neuronal survival and differentiation in distinct subpopulations, probably in combination with other factor(s). Our results indicate that $\mathrm{Hh}$ regulates the proportion of gata3 neurons within the neuroD+ population of the SAG. In line with this idea, a recent paper shows that hip, an inhibitor of $\mathrm{Hh}$ targets that depends on Hh signaling, is only expressed in a subpopulation of SAG neurons (Hammond and Whitfield, 2009). An explanation for our results would be that in the absence of Hh signaling, part of the SAG neurons improperly expresses gata3 and the resulting mispecified neurons are eliminated by apoptosis.

\section{Anteroposterior segregation of neurons within the SAG correlates with topographic position of the sensory epithelia within the otic vesicle}

Tracing of afferent neurons specifically innervating each macula revealed that these two neuronal populations are segregated within the SAG. Moreover, our data indicate that the anterior part of the SAG contains neurons innervating the anterior macula (AM) and the anterior and lateral cristae (AC and LC), whereas the posterior part is composed of otic neurons for posterior macula (PM) and posterior crista (PC). However, colabeling of these two neuronal populations after DiI-application in the PM was not expected, as the information for macular (linear acceleration) and canal (angular acceleration) organs must be conducted by different neurons. Although we cannot exclude that colabeling of PM and PC neurons results from contamination during injection, due to the location of the neuronal fibers immediately beneath the injected epithelium, DiI injection in the posterior part of the SAG results in labeling of peripheral processes directed towards both PM and PC. This clearly demonstrates that neurons for PM and PC at least overlap in the posterior part of the SAG and are completely segregated from neurons innervating AM, $\mathrm{AC}$ and LC, located in the anterior part of the SAG. Interestingly, in mammals, posterior canal and saccular neurons seem to overlap in one part of the vestibular ganglion that is devoid of other endorganspecific neurons (Maklad and Fritzsch, 1999).

Dye-tracing experiments in NeuroD-null mice showed an important reduction of otic endorgan innervation; however, while innervation to anterior and lateral cristae is almost unaffected, it is moderately reduced to the utricule, and drastically reduced to the cochlea, saccular macula and posterior crista (Kim et al., 2001). This anterior to posterior gradient of fiber loss suggests a developmental relationship between the formation of the innervation towards all the posterior sensory epithelia, either vestibular or auditory. Fate-mapping studies in chick, suggest not only that a part of the territory giving rise to the maculae overlaps with the precursors of the basilar papilla and the posterior crista, but also that sensory cells for one endorgan and their innervating neurons arise in close proximity (Bell et al., 2008). Additional data also point to a close relation between the development of PC and PM innervations, despite the functional specialization of their corresponding sensory epithelia: central projections for PC and PM neurons initially overlap largely but progressively segregate to their respective discrete projection areas during development (Maklad et al., 2004). 
Indeed, we showed here that the organization in sharply segregated SAG neuronal populations and areas of central projections does not reflect the two functional specifications of vestibular endorgans (angular acceleration from the cristae and linear acceleration from the maculae) but likely reflects the anterior or posterior positions of their corresponding sensory epithelia within the inner ear. The vestibular nerve may have initially been composed of two distinct nerves, anterior and posterior (Fritzsch, 1998), what may account for the sharp segregation visible in the developing SAG of zebrafish.

\section{Does $\mathrm{Hh}$ act as a major driving force in inner ear evolution?} Evolution has transformed a simple ear with only two cristae and a single macula found in agnathes, in a complex structure containing up to nine distinct endorgans in derived vertebrates (Lewis et al., 1985). It has been proposed that evolution of the inner ear relies on the progressive division of a given sensory patch. The newly generated patch will acquire specific anatomical and functional features to detect previously unexplored characteristics of the mechanical stimuli, and novel associated neurons will form (Fritzsch et al., 2002). In line with this hypothesis, it was proposed that during evolution, saccula splits to give rise to the basilar papilla in birds and the cochlea in mammals (Fritzsch, 1987). Experimental evidences also suggest a recapitulation of this evolutionary process during the development of the vertebrate inner ear, since in mouse sensory epithelia appear sequentially: the utricule is formed before the saccule, followed by the formation of the cochlea. Our study also highlights an asynchronic development of AM versus PM in zebrafish. Concerning the role of Hh signaling in the inner ear development and evolution, it seems relevant that in zebrafish, Hh pathway is involved both in the formation of the sensory patch for auditory function, and of its discrete innervation pattern. Previous studies showed that $\mathrm{Hh}$ is required for the formation of the basilar papilla in chick (Bok et al., 2005) and of the cochlea in mouse (Riccomagno et al., 2002). Otic neuron production is compromised in $\mathrm{Shh}^{-1-}$ mice (Riccomagno et al., 2002), but the aspect of endorgan-specific innervation was not investigated. With the cochlea viewed as an outgrowth of the saccule, a primary effect of Hh signaling inactivation in the formation of the saccular macula would impair further development of the auditory endorgan in mouse and chick. Indeed, $S h h^{-\prime-}$ mice display a single domain of expression of a marker for utricular, saccular and cochlear epithelia (Riccomagno et al., 2002), a defect comparable to the fusion of macular sensory epithelia described in the zebrafish $h$ h mutants (Hammond et al., 2003) and in the present study. It is not clear whether Hh acts directly on the separation of an initial sensory domain in different sensory epithelia, as in lamprey a single macula communis coexists with apparently active $\mathrm{Hh}$ signaling in the otic epithelium (Hammond and Whitfield, 2006). However, it is interesting to note that within this macula communis, anterior and posterior hair cells already develop asynchronously and asymmetrically (Hammond and Whitfield, 2006). Finally, after emergence of the cochlea during evolution, Shh pathway has been involved in the specification, location and size of the prosensory domain, changes that are thought to correlate with the ability to perceive high frequencies in mammals (Driver et al., 2008). We propose that Hh pathway first directed the formation of a second functionally relevant sensory patch and then has been probably continuously recruited to direct the development of this organ, and of the derived ones, toward an auditory fate.

\section{References}

Andermann P, Ungos J, Raible DW (2002) Neurogenin1 defines zebrafish cranial sensory ganglia precursors. Dev Biol 251:45-58.

Bell D, Streit A, Gorospe I, Varela-Nieto I, Alsina B, Giraldez F (2008) Spatial and temporal segregation of auditory and vestibular neurons in the otic placode. Dev Biol 322:109-120.

Bermingham NA, Hassan BA, Price SD, Vollrath MA, Ben-Arie N, Eatock RA, Bellen HJ, Lysakowski A, Zoghbi HY (1999) Math1: an essential gene for the generation of inner ear hair cells. Science 284:1837-1841.

Bever MM, Fekete DM (1999) Ventromedial focus of cell death is absent during development of Xenopus and zebrafish inner ears. J Neurocytol 28:781-793.

Bok J, Bronner-Fraser M, Wu DK (2005) Role of the hindbrain in dorsoventral but not anteroposterior axial specification of the inner ear. Development 132:2115-2124.

Chen JK, Taipale J, Cooper MK, Beachy PA (2002) Inhibition of hedgehog signaling by direct binding of cyclopamine to smoothened. Genes Dev 16:2743-2748.

Chen W, Burgess S, Hopkins N (2001) Analysis of the zebrafish smoothened mutant reveals conserved and divergent functions of hedgehog activity. Development 128:2385-2396.

Cheng CW, Yan CH, Choy SW, Hui MN, Hui CC, Cheng SH (2007) Zebrafish homologue irxla is required for the differentiation of serotonergic neurons. Dev Dyn 236:2661-2667.

Cole LK, Ross LS (2001) Apoptosis in the developing zebrafish embryo. Dev Biol 240:123-142.

D’Amico-Martel A (1982) Temporal patterns of neurogenesis in avian cranial sensory and autonomic ganglia. Am J Anat 163:351-372.

Driver EC, Pryor SP, Hill P, Turner J, Rüther U, Biesecker LG, Griffith AJ, Kelley MW (2008) Hedgehog signaling regulates sensory cell formation and auditory function in mice and humans. J Neurosci 28:7350-7358.

Fariñas I, Jones KR, Tessarollo L, Vigers AJ, Huang E, Kirstein M, de Caprona DC, Coppola V, Backus C, Reichardt LF, Fritzsch B (2001) Spatial shaping of cochlear innervation by temporally regulated neurotrophin expression. J Neurosci 21:6170-6180.

Fritzsch B (1987) Inner ear of coelacanth fish Latimeria has tetrapod affinities. Nature 237:153-154.

Fritzsch B (1998) Evolution of the vestibulo-ocular system. Otolaryngol Head Neck Surg 119:182-192.

Fritzsch B, Beisel KW (2004) Keeping sensory cells and evolving neurons to connect them to the brain: molecular conservation and novelties in vertebrate ear development. Brain Behav Evol 64:182-197.

Fritzsch B, Beisel KW, Bermingham NA (2000) Developmental evolutionary biology of the vertebrate ear: Conserving mechanoelectric transduction and developmental pathways in diverging morphologies. Neuroreport 11:R35-R44.

Fritzsch B, Beisel KW, Jones K, Fariñas I, Maklad A, Lee J, Reichardt LF (2002) Development and evolution of inner ear sensory epithelia and their innervation. J Neurobiol 53:143-156.

Fritzsch B, Matei VA, Nichols DH, Bermingham N, Jones K, Beisel KW, Wang VY (2005) Atoh1 null mice show directed afferent fiber growth to undifferentiated ear sensory epithelia followed by incomplete fiber retention. Dev Dyn 233:570-583.

Fritzsch B, Beisel KW, Hansen LA (2006) The molecular basis of neurosensory cell formation in ear development: a blueprint for hair cell and sensory neuron regeneration? Bioessays 28:1181-1193.

Haddon C, Lewis J (1996) Early ear development in the embryo of the zebrafish, Danio rerio. J Comp Neurol 365:113-128.

Haddon C, Jiang YJ, Smithers L, Lewis J (1998) Delta-Notch signalling and the patterning of sensory cell differentiation in the zebrafish ear: evidence from the mind bomb mutant. Development 125:4637-4644.

Hammond KL, Whitfield TT (2006) The developing lamprey ear closely resembles the zebrafish otic vesicle: otx1 expression can account for all major patterning differences. Development 133:1347-1357.

Hammond KL, Whitfield TT (2009) Expression of zebrafish hip: Response to Hedgehog signalling, comparison with ptcl expression, and possible role in otic patterning. Gene Expr Patterns 9:391-396.

Hammond KL, Loynes HE, Folarin AA, Smith J, Whitfield TT (2003) Hedgehog signalling is required for correct anteroposterior patterning of the zebrafish otic vesicle. Development 130:1403-1417.

Hauptmann G, Gerster T (1994) 2-color whole-mount in-situ hybridization to vertebrate and Drosophila embryos. Trends Genet 10:266. 
Itoh M, Chitnis AB (2001) Expression of proneural and neurogenic genes in the zebrafish lateral line primordium correlates with selection of hair cell fate in neuromasts. Mech Dev 102:263-266.

Karis A, Pata I, van Doorninck JH, Grosveld F, de Zeeuw CI, de Caprona D, Fritzsch B (2001) Transcription factor GATA-3 alters pathway selection of olivocochlear neurons and affects morphogenesis of the ear. J Comp Neurol 429:615-630.

Kim WY, Fritzsch B, Serls A, Bakel LA, Huang EJ, Reichardt LF, Barth DS, Lee JE (2001) NeuroD-null mice are deaf due to a severe loss of the inner ear sensory neurons during development. Development 128:417-426.

Koundakjian EJ, Appler JL, Goodrich LV (2007) Auditory neurons make stereotyped wiring decisions before maturation of their targets. J Neurosci 27:14078-14088.

Kwak SJ, Vemaraju S, Moorman SJ, Zeddies D, Popper AN, Riley BB (2006) Zebrafish pax5 regulates development of the utricular macula and vestibular function. Dev Dyn 235:3026-3038.

Lawoko-Kerali G, Rivolta MN, Lawlor P, Cacciabue-Rivolta DI, LangtonHewer C, van Doorninck JH, Holley MC (2004) GATA3 and NeuroD distinguish auditory and vestibular neurons during development of the mammalian inner ear. Mech Dev 121:287-299.

Lecaudey V, Ulloa E, Anselme I, Stedman A, Schneider-Maunoury S, Pujades C (2007) Role of the hindbrain in patterning the otic vesicle: a study of the zebrafish vhnf1 mutant. Dev Biol 303:134-143.

Lee JE, Hollenberg SM, Snider L, Turner DL, Lipnick N, Weintraub H (1995) Conversion of Xenopus ectoderm into neurons by NeuroD, a basic helixloop-helix protein. Science 268:836-844.

Lewis ER, Leverenz EL, Bialek WS (1985) The vertebrate inner ear, pp 248. Boca Raton, FL: CRC.

Ma Q, Chen Z, del Barco Barrantes I, de la Pompa JL, Anderson DJ (1998) Neurogenin 1 is essential for the determination of neuronal precursors for proximal cranial sensory ganglia. Neuron 20:469-482.

Maklad A, Fritzsch B (1999) Incomplete segregation of endorgan-specific vestibular ganglion cells in mice and rats. J Vestib Res 9:387-399.

Maklad A, Fritzsch B, Hansen LA (2004) Development of vestibular afferent projections into the hindbrain and their central targets. Cell Tissue Res 315:167-180.

Millimaki BB, Sweet EM, Dhason MS, Riley BB (2007) Zebrafish atoh1 genes: classic proneural activity in the inner ear and regulation by $f g f$ and notch. Development 134:295-305.

Park SH, Yeo SY, Yoo KW, Hong SK, Lee S, Rhee M, Chitnis AB, Kim CH (2003) Zath3, a neural basic helix-loop-helix gene, regulates early neurogenesis in the zebrafish. Biochem Biophys Res Commun 308:184-190.

Pittman AJ, Law MY, Chien CB (2008) Pathfinding in a large vertebrate axon tract: isotypic interactions guide retinotectal axons at multiple choice points. Development 135:2865-2871.

Raft S, Koundakjian EJ, Quinones H, Jayasena CS, Goodrich LV, Johnson JE, Segil N, Groves AK (2007) Cross-regulation of Ngn1 and Math1 coordinates the production of neurons and sensory hair cells during inner ear development. Development 134:4405-4415.

Riccomagno MM, Martinu L, Mulheisen M, Wu DK, Epstein DJ (2002) Specification of the mammalian cochlea is dependent on sonic hedgehog. Genes Dev 16:2365-2378.

Riley BB, Phillips BT (2003) Ringing in the new ear: resolution of cell interactions in otic development. Dev Biol 261:289-312.

Riley BB, Zhu C, Janetopoulos C, Aufderheide KJ (1997) A critical period of ear development controlled by distinct populations of ciliated cells in the zebrafish. Dev Biol 191:191-201.

Sun Z, Hopkins N (2001) vhnf1, the MODY5 and familial GCKDassociated gene, regulates regional specification of the zebrafish gut, pronephros, and hindbrain. Genes Dev 15:3217-3229.

Takebayashi K, Takahashi S, Yokota C, Tsuda H, Nakanishi S, Asashima M, Kageyama R (1997) Conversion of ectoderm into a neural fate by ATH-3, a vertebrate basic helix-loop-helix gene homologous to Drosophila proneural gene atonal. EMBO J 16:384-395.

Torres M, Giráldez F (1998) The development of the vertebrate inner ear. Mech Dev 71:5-21.

Vanderlaan G, Tyurina OV, Karlstrom RO, Chandrasekhar A (2005) Gli function is essential for motor neuron induction in zebrafish. Dev Biol 282:550-570.

Whitfield TT, Riley BB, Chiang MY, Phillips B (2002) Development of the zebrafish inner ear. Dev Dyn 223:427-458.

Xiao T, Roeser T, Staub W, Baier H (2005) A GFP-based genetic screen reveals mutations that disrupt the architecture of the zebrafish retinotectal projection. Development 132:2955-2967. 